\title{
Isolation of Biosurfactant Producing Bacteria from Garbage Soil
}

\author{
M. Rajesh, M. Samundeeswari, B. Archana* \\ Department of Zoology, The American College, Madurai, Tamil Nadu, India \\ *Corresponding author: archanaabalan18@gmail.com
}

\begin{abstract}
Biosurfactants are amphiphilic compounds secreted extra-cellularly by microorganisms. Biosurfactants contains hydrophobic and hydrophilic moieties that reduce the surface tension and interfacial tension by accumulating at the interface of hydrophobic substances and thus increase the solubility, bioavailability and subsequent biodegradation of the hydrophobic or insoluble organic compounds. The present study focused on the isolation of biosurfactant producing microorganisms from garbage soil. Isolated microorganisms were identified based on the biochemical characterization as Staphylococcus aureus and Bacillus cereus. Qualitative analysis of biosurfactant production was identified by performing assays like hemolysis on blood agar, penetration assay and $\mathrm{C}-\mathrm{TAB}$ agar assay. The present study confirms that isolated microorganisms have biosurfactant property.
\end{abstract}

Keywords: biosurfactant, staphylococcus aureus, bacillus cereus

Cite This Article: M. Rajesh, M. Samundeeswari, and B. Archana, "Isolation of Biosurfactant Producing Bacteria from Garbage Soil.” Journal of Applied \& Environmental Microbiology, vol. 5, no. 2 (2017): 74-78. doi: 10.12691/jaem-5-2-3.

\section{Introduction}

Biosurfactanats are a heterogenous group of surface active molecules secreted extracellularly or adhere to the cell membrane by several bacterial and fungal species [2]. These naturally occurring surface active molecules are amphiphilic in nature, it contains hydrophilic and hydrophobic moieties which reduces the surface and interfacial tension with high specificity, low toxicity and biodegradability. This facilitates properties like emulsification, foaming, detergency and dispersing.[9]. There are high molecular weight biosurfactants which contain polysaccharides and proteins and low molecular weight biosurfactants which contain glycolipids and lipopeptides. Based on their charges they are classified as anionic, cationic and non-ionic biosurfactants [10]. It enhances the growth of microorganisms by increasing the availability of hydrophobic nutrients [4] and converting it into harmless products [11]. By the unique property of reducing the surface tension, it increases the area of contact of insoluble compounds and enhances the mobility, bioavailability and biodegradation of hydrophobic contaminant [7]. Biosurfactants are highly advantageous to their producing strains, by enhancing the bioavailability of hydrophobic contaminants, bind to heavy metals, possess antimicrobial activity, regulate the attachment or detachment of microorganisms to and from surfaces [12].

Biosurfactants are superior over chemical surfactants with its simple chemical structure, higher stability, high foaming capacity, low toxicity, $\mathrm{pH}$, environmental compatibility, and biodegradability. Biosurfactants they have specific functional groups which provide specificity in detoxification of specific pollutants under extreme salinity, temperature and $\mathrm{pH}$ [9].Their properties like low toxicity and eco-friendly nature contribute to the applicability of biosurfactants in cosmetic, pharmaceutical, oil and food industries [7]. The present study is to isolate microorganisms from garbage soil and qualitative analysis of its biosurfactant production.

\section{Materials and Methods}

\subsection{Sample Collection}

Garbage soil sample (waste disposable sites dumped with polythene bags and plastic cups) was collected from THE AMERICAN COLLEGE campus, Madurai, Tamil Nadu, India. The sample was collected at the depth of 3$5 \mathrm{~cm}$, in a sterile container and then air dried at room temperature [Figure 1].

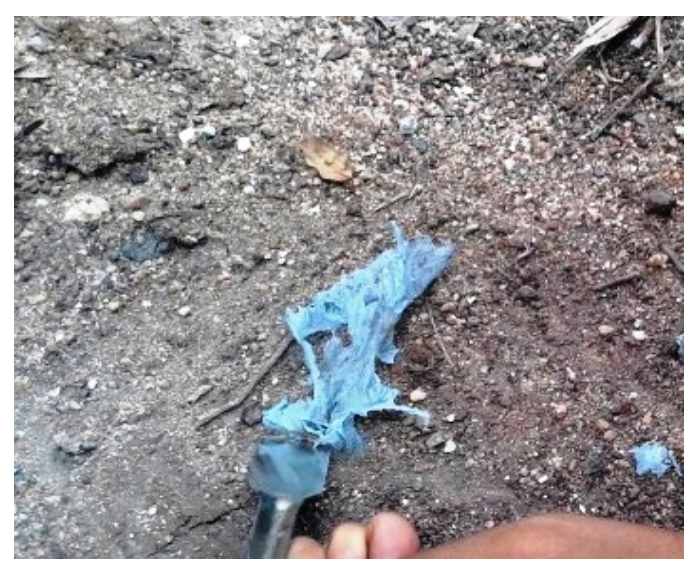

Figure 1. Collection of garbage soil sample 


\subsection{Identification of Microorganisms}

Isolated organisms were identified based on their morphology, cultural and biochemical characteristics. All the isolates were subjected to Gram staining, Mannitol fermentation test on Mannitol-Salt agar, hemolytic activity on blood agar and catalase tests were performed. Species level of identification was done by biochemical analysis in the Bose clinical laboratory in Simmakal, Madurai, Tamil Nadu 625 001, India.

\subsection{Screening of Biosurfactant Production}

\subsubsection{Culture Medium and Bacterial Growth}

Bacteria were grown aerobically in $500 \mathrm{ml}$ Erlenmeyer flask with $100 \mathrm{ml}$ of mineral salt medium and crude oil $(1.0 \%, \mathrm{w} / \mathrm{v})$. Flasks containing sterilized mineral salt medium were inoculated with a loop full of bacterial culture and the culture flasks were maintained in a shaker for 7 days at $200 \mathrm{RPM}$ and $30^{\circ} \mathrm{C}$. After 7 days of incubation, culture broth from each flask was centrifuged at $6000 \mathrm{rpm}$ at $4^{\circ} \mathrm{C}$ for 15 minutes and the supernatant was filtered through $0.45 \mu \mathrm{m}$ pore size filter paper (Millipore). This cell-free culture broth was used for further assays.

\subsubsection{Hemolytic Activity}

A Hemolytic assay was performed in 5\% sheep blood agar plates. $50 \mu \mathrm{l}$ of bacterial culture grown in a mineral salt medium was spot inoculated onto blood agar plates and incubated for $48 \mathrm{~h}$ at $37^{\circ} \mathrm{C}$. The plates were visually inspected for a clear zone (hemolysis) around the colony. The diameter of the clear zone is a qualitative method used as an indicator of biosurfactant production.

\subsection{3. cTAB Agar Plate}

Isolated microorganism was plated on mineral salt medium containing cationic surfactant cetyltrimethyl ammonium bromide (cTAB) and the basic dye methylene blue. If anionic surfactants are secreted by the microbes growing on the plate, they form a dark blue, insoluble ion pair with cetyltrimethylammonium bromide and methylene blue. Thus, biosurfactant producing colonies are surrounded by a dark blue ring.

\subsubsection{Penetration Assay}

For penetration assay, hydrophobic paste consisting of oil and silica gel was prepared and $150 \mu$ l of this paste was added to each well of microtitre plates. Add $90 \mu \mathrm{l}$ of culture supernatant and $10 \mu \mathrm{l}$ of red staining solution like saffranin on the top of the paste. If the supernatant contains biosurfactant it crosses the oil barrier and enters into the paste. The upper phase changes from red to cloudy white within few minutes [12].

\section{Results}

\subsection{Isolation of Microorganisms from Garbage Soil}

Two different microorganisms were isolated from garbage soil and identified based on the biochemical characterization.

\subsection{Identification of Microorganisms}

Biochemical characterization such as Gram staining, Mannitol fermentation test on M.S agar, hemolytic activity on blood agar and Catalase tests were performed. As per results, such as Gram staining [Figure 2 A], Hemolysis on blood agar [Figure 5 A], Mannitol fermentation on Mannitol salt agar medium [Figure 3] and positive catalase reaction [Figure $4 \mathrm{~A}$ ] indicates that the isolated organism was Staphylococcus sp [5]. Similarly, the second organism was identified as Bacillus sp [1] based on Gram staining [Figure 2 B] Creamy white larger colonies with more mucoid [Figure 6] and partial hemolysis on blood agar [Figure $5 \mathrm{~B}$ ] [1] and positive Catalase reaction [Figure $4 \mathrm{~B}$ ].

For species level identification several biochemical tests were done in the Bose clinical laboratory in simmakkal. Staphylococcus sp showed a positive result for tests includes urea hydrolysis test, B-methyl glucoside utilization test and fermentation of glucose glycerol, sucrose, mannitol and maltose. And negative results for aesculin hydrolysis test, arginine utilization test and fermentation of sorbitol, rhamnose and trehalose. Whereas, Bacillus sp, showed a positive results for urea hydrolysis test, B-methyl glucoside utilization test and fermentation of glucose, glycerol, sucrose, mannitol and maltose. And negative results for aesculin hydrolysis test, arginine utilization test and fermentation of sorbitol, rhamnose and trehalose. Thus based on biochemical characterization, the isolated organisms were identified as Staphylococcus aureus [Table 1] and Bacillus cereus [Table 2].

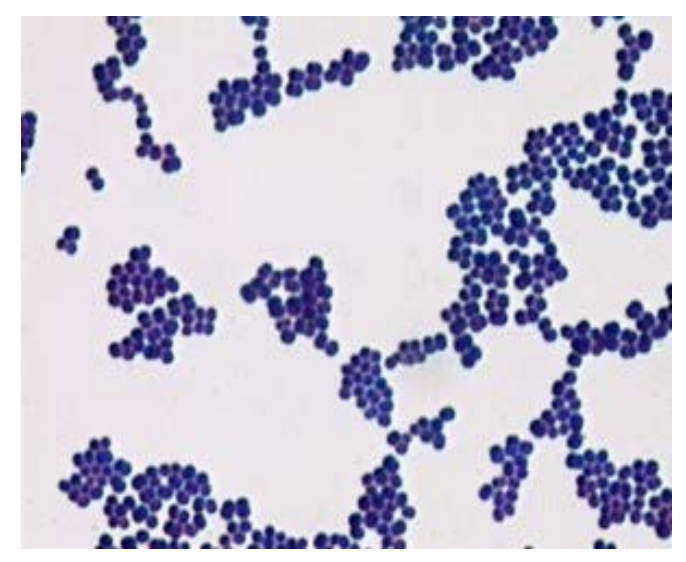

Figure 2A. Gram staining of S. aureus

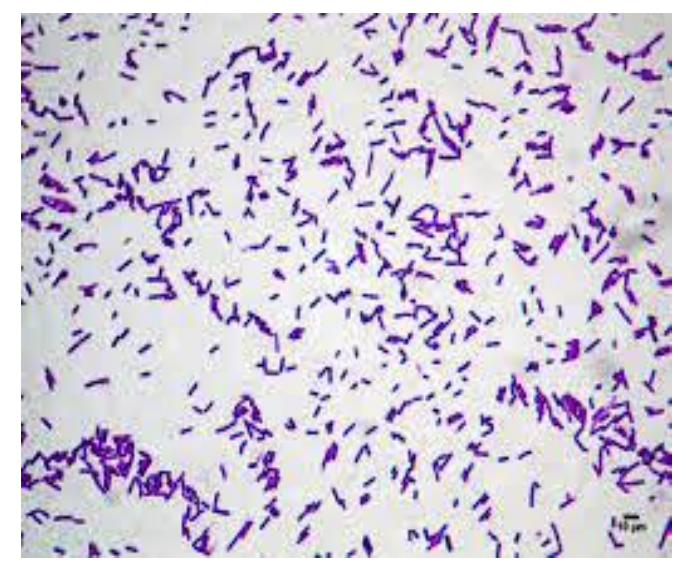

Figure 2B. Grams staining of Bacillus cereus 


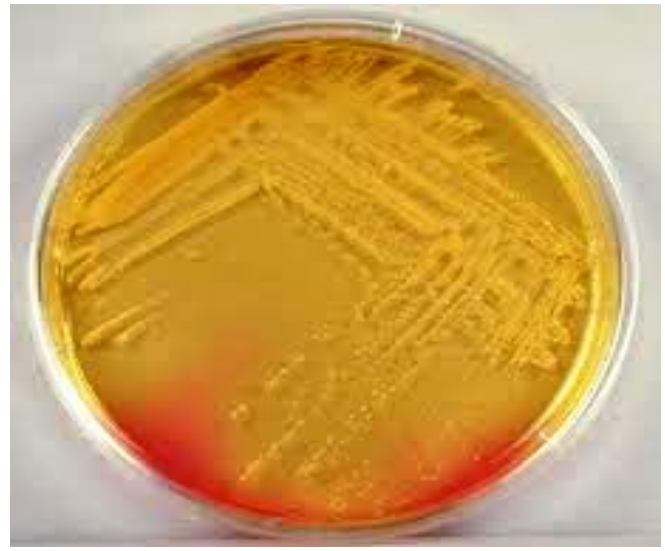

Figure 3. Mannitol fermentation test for S.aureus

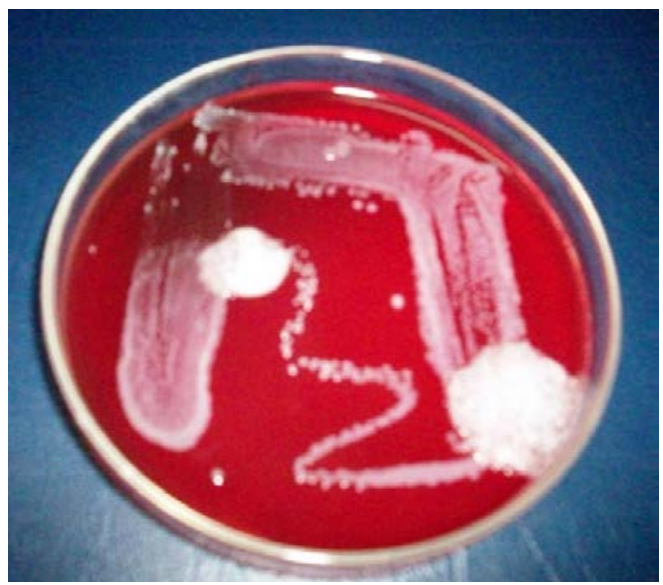

Figure 4A. Catalase test for S.aureus

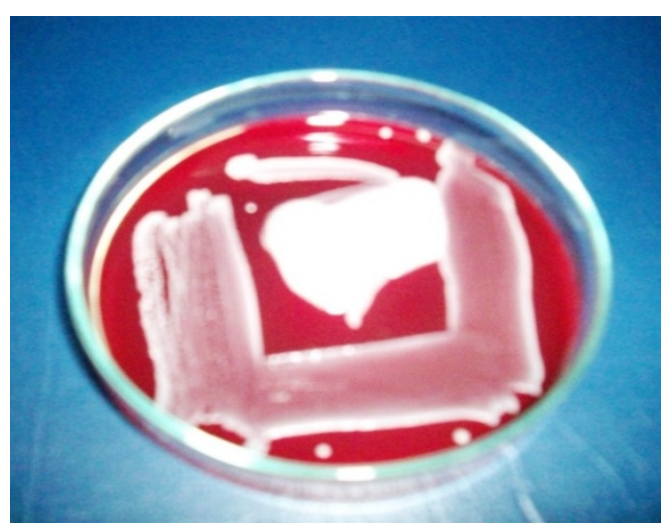

Figure 4B. Catalase for B.cereus

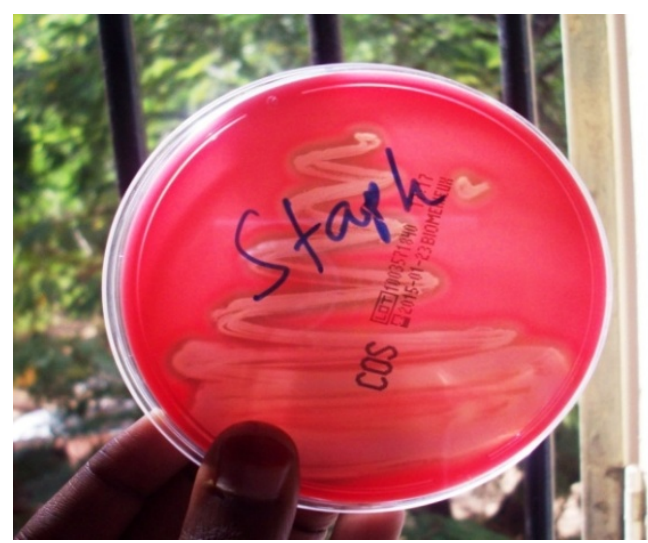

Figure 5A. Hemolysis test for S.aureus

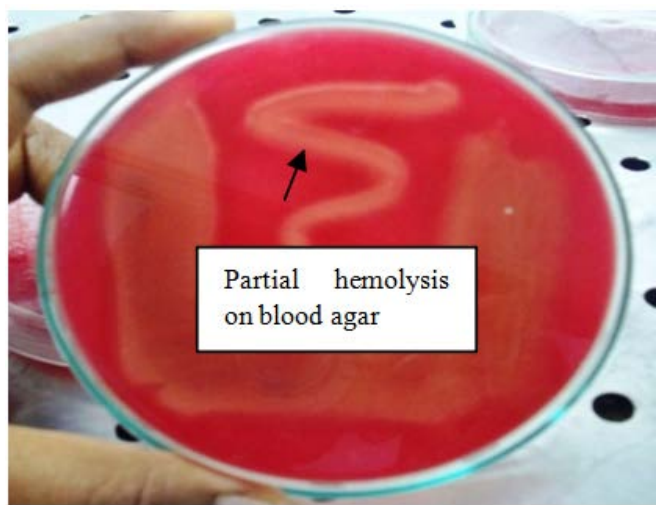

Figure 5B. Hemolysis test for B.cereus

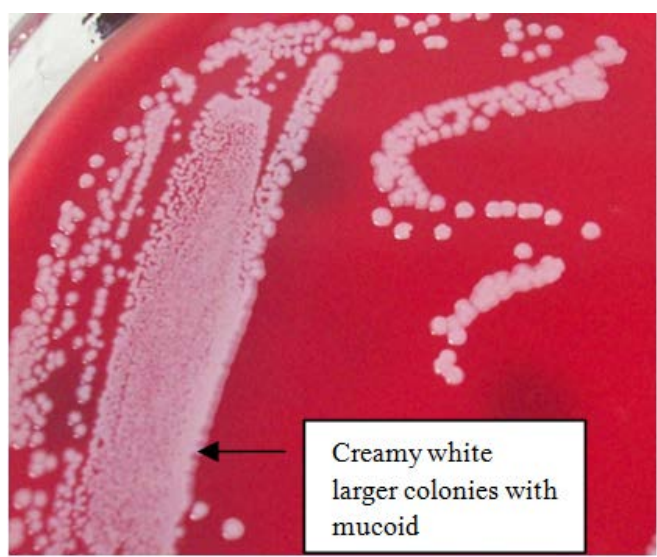

Figure 6. Creamy white coloies of B.cereus on blood agar

Table 1. Biochemical tests for staphylococcus aureus

\begin{tabular}{|l|l|l|}
\hline S.No & Biochemical Tests & Results \\
\hline 1 & Urea & Positive \\
\hline 2 & Sorbitol & Negative \\
\hline 3 & Aesculin & Negative \\
\hline 4 & Rhamnose & Negative \\
\hline 5 & Trehalose & Negative \\
\hline 6 & Glucose & Positive \\
\hline 7 & B-methyl glucoside & Positive \\
\hline 8 & Arginine & Negative \\
\hline 9 & Glycerol & Positive \\
\hline 10 & Sucrose & Positive \\
\hline 11 & Mannitol & Positive \\
\hline 12 & Maltose & Positive \\
\hline
\end{tabular}

Table 2. Biochemical tests for Bacillus cereus

\begin{tabular}{|l|l|l|}
\hline S.No & Biochemical tests & Results \\
\hline 1 & Urea & Positive \\
\hline 2 & Sorbitol & Negative \\
\hline 3 & Aesculin & Negative \\
\hline 4 & Rhamnose & Negative \\
\hline 5 & Trehalose & Negative \\
\hline 6 & Glucose & Positive \\
\hline 7 & B-methyl glucoside & Positive \\
\hline 8 & Arginine & Negative \\
\hline 9 & Glycerol & Positive \\
\hline 10 & Sucrose & Positive \\
\hline 11 & Mannitol & Positive \\
\hline 12 & Maltose & Positive \\
\hline
\end{tabular}




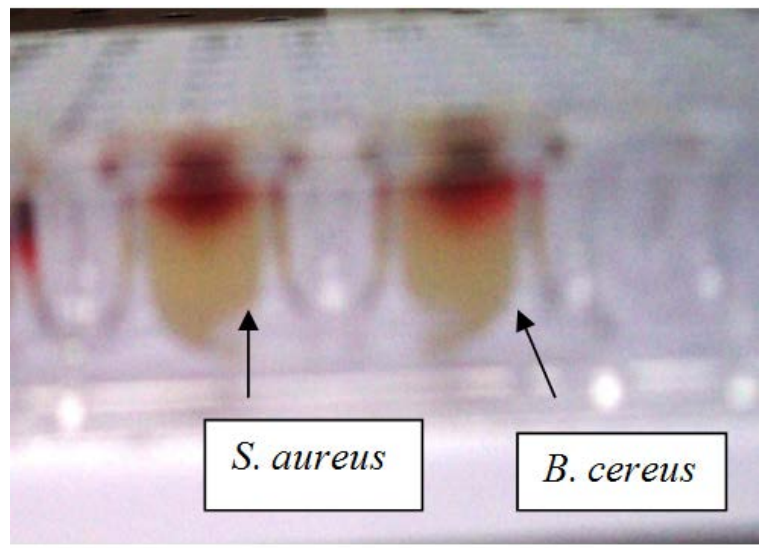

Figure 7A. Penetration assay-Before incubation

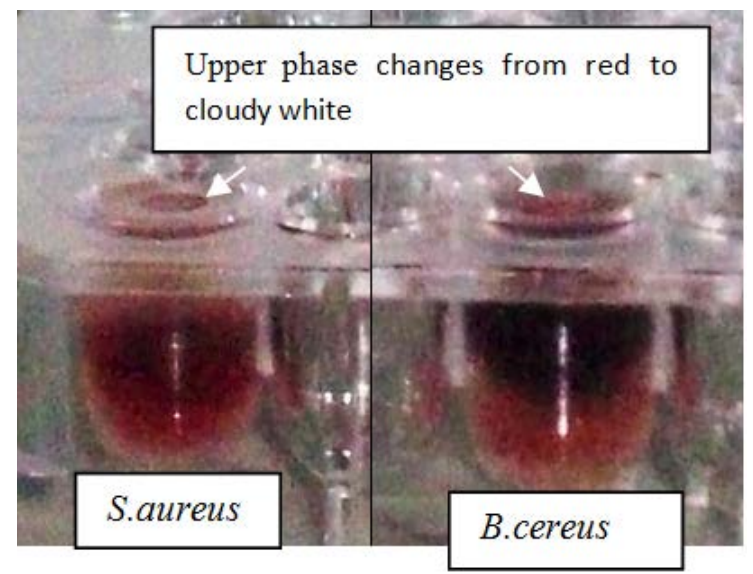

Figure 7B. Penetration assay - After incubation

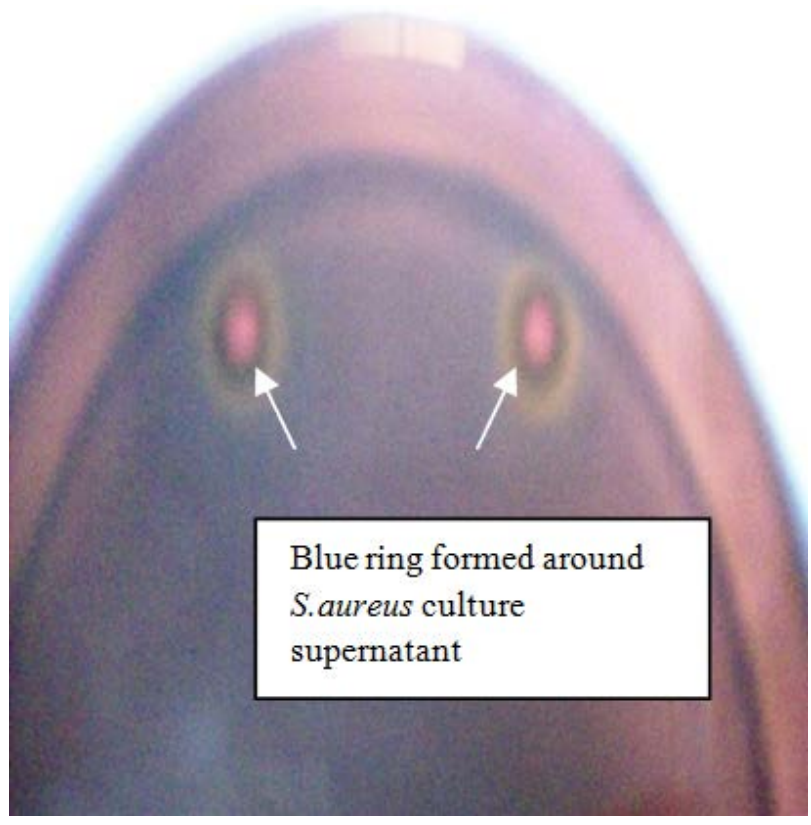

Figure 8A. C-TAB agar plate - S.aureus

\subsection{Screening of Biosurfactant Production}

Qualitative analysis of biosurfactant production was achieved by performing Hemolysis on blood agar, Penetration assay, and CTAB agar plate assay. Biosurfactant production by Staphylococcus aureus and Bacillus cereus was identified based on results such as Hemolysis on blood agar [Figure $5 \mathrm{~A}$ and B], Biosurfactants producing organisms cause lysis of erythrocytes and show a transparent zone around the colonies. Penetration of red dye in penetration assay [Figure $7 \mathrm{~A}$ and B] Biosurfactant present in the culture supernatant degrades the oil barrier and allow the red dye to enter into silica gel and the upper phase turns into cloudy white .Blue ring formation on CTAB agar [Figure $8 \mathrm{~A}$ and B]. Anionic biosurfactants interacts with $\mathrm{CTAB}$ and Methylene blue and forms a insoluble ion pair which leads to the formation of blue ring [12].

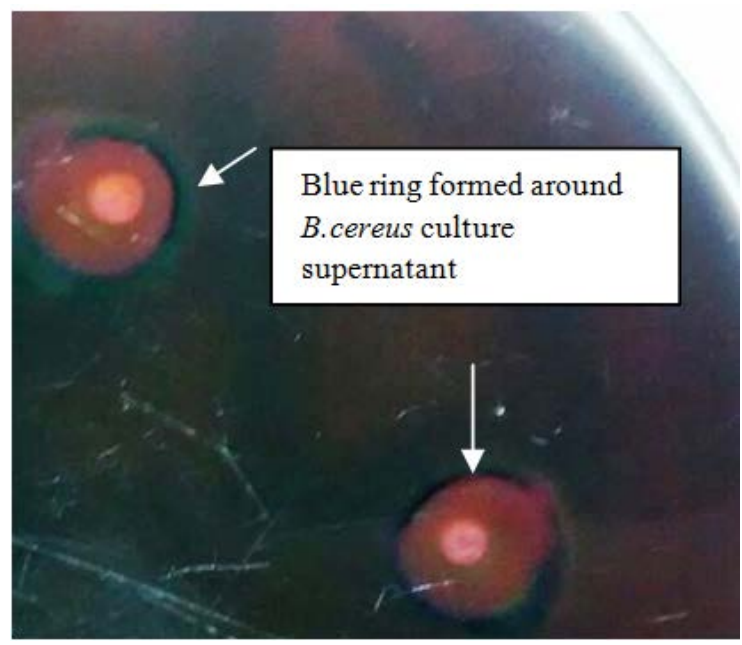

Figure 8B. C-TAB agar plate - B.cereus

\section{Discussion}

Biosurfactants are widely used in hydrocarbon bioremediation field since they can enhance the growth on hydrophobic surface and can increase the nutrient uptake of hydrophobic substrates, thereby overcoming the poor availability of hydrocarbon contaminants to microorganisms [3]. Microorganisms having diverse synthetic capabilities, offer potential in expanding the range of surfactant with novel compounds, thereby providing new possible applications. Biosurfactants are ecologically acceptable than chemical surfactants. The absence of degrading capability could be related to the lack of biosurfactant production.

In the present study, two different bacterial species were isolated from garbage soil. The identification of the isolated strains was done by gram staining, mannitol fermentation, catalase test etc. From the results, these organisms were identified as Bacillus cereus and Staphylococcus aureus. In case of qualitative analysis of biosurfactant production hemolytic activity was observed in both the isolated microorganisms because biosurfactants can cause lysis of erythrocytes, Dark blue ring formation on CTAB agar and in penetration assay, penetration of red dye into silica gel turns the coloured supernatant into cloudy white which indicates the production of biosurfactant [12]. Thus above experiments confirm the presence of biosurfactant production by both the isolated Staphylococcus sp. and Bacillus sp. The use of biosurfactant has been found to enhance the degradation in various cases. Biosurfactants because of their inherent degradability they have potential application in biodegradation of hydrophobic contaminants, waste water treatment and bioremediation processes $[6,8]$. 


\section{Conclusion}

In this study, Staphylococcus aureus and Bacillus cereus were identified as biosurfactant producers and can be used in bioremediation technologies and other industrial processes.

\section{References}

[1] Al-Bager. S.D (2005). Bacillus cereus - King Saud University, College of Pharmacy, Department of Pharmaceutics, Section of Microbiology.

[2] Anandara.B and P. Thivakaran (2010). Isolation and production of Biosurfactant producing organism from oil spilled soil, Journal of Biological science and Technology volume 1, ISSN: 120-126.

[3] Chandran . P abd Nilanjana das (2010). Biosurfactant and diesel oil degradation by yeast species, Trichosporon asahii isolated from petroleoum hydrocarbon contaminated soil.International Journa of Engineering Science and Technology Vol. 2(12), 6942-6953.

[4] Kiran G.S, Hema. T.A, Gandhimathi.R, Joseph selvin, Anto Thomas. T, Rajeetharavji. T and Natarajaseenivasana .K (2009), Optimization and production of a biosurfactant from sponge associated marine fungus Aspergillusustus MSF3. Colloids surface. B., 73(2), 250-256
[5] Li Zhu.L, Feng-cai Zou, Yu-lin Yan, Qi-hui Wang, Yong-qiang Shi, and Wei-jie Qu (2016), The Characteristics of Staphylococcus aureus Small Colony Variant Isolated from Chronic Mastitis at a Dairy Farm in Yunnan Province, China. The Scientific World Journal Volume 2016, Article ID 9157605, 8 pages.

[6] Muller-Hunting. R, Wagner. F, Blaszczyk. R, Kosaric. N (1993), Biosurfactants production, properties, application. Kosaric $\mathrm{N}$ ed. 447-469.

[7] Rita de Cássia F. S. Silva, Darne G. Almeida, Raquel D. Rufino, Juliana M. Luna, Valdemir A. Santos and Leonie Asfora Sarubbo (2014), Applications of Biosurfactants in petroleum industry and the remediation of oil spills. International journal of molecular science- ISSN 1422-0067.

[8] Sim.L, Ward. O.P, Lizy (1997). Production and characterization of biosurfactant isolated from Pseudomonas aeroginousa UW-1. Journal of Industrial Microbiology and Biotechnology.19, 232-236.

[9] Sneha-Chakrabarti (2013). Bacterial Biosurfactant: Characterization, Antimicrobial and metal remediation properties.

[10] Varjani. J.S, D. P. Rana, S. Bateja, M. C. Sharma and V. N. Upasani (2014). Screening and identification of biosurfactant (bioemulsifier) producing bacteria from crude oil contaminated sites of Gujarat, India. International Journal of Innovative Research in Science, Engineering and Technology - Vol. 3, Issue 2.

[11] Vijaya. B, N.R. Jayalakshmi and K. Manjunath (2013). Enumeration of biosurfactant producing microorganisms from oil contaminated soil in and around Bangalore (India). International journal of current science, 5: 86-94.

[12] Walter, Christoph Syldatk and Rudolf Hausmann, (2010). Screening Concepts for the Isolation of Biosurfactant Producing Microorganisms. 